\title{
Sources of Blood Glucose in the Rat Fetus
}

\author{
Emilio Bossi ${ }^{[21]}$ and Robert E. Greenberg \\ Department of Pediatrics, Stanford University School of Medicine, Stanford, California, USA, and \\ Department of Pediatrics, University of Berne, Berne, Switzerland
}

\section{Extract}

Pregnant Sprague-Dawley rats from the 18th to the 21 st day of gestation were fasted for $16 \mathrm{hr}$ and anesthetized, and a constant infusion of uniformly labeled glucose- ${ }^{14} \mathrm{C}$ and of glucose- $2{ }^{3} \mathrm{H}$ was started. The purpose was to see if the regulation of fetal blood glucose concentration differed from the maternal during fasting. The results are given in Figures 2 and 3. During the course of the infusions, the specific activity of glucose was, at all points measured, two to four times lower in the fetal than in the maternal serum. The higher fetal than maternal supply of blood glucose could be derived either frcm gluconeogenesis or from glycogenolysis in the fetus.

In vitro studies indicate that the fetal rat liver is unable to perform gluconecgenesis. Therefore, the placenta was investigated for the presence of a gluconeogenetic pathway. Placenta slices showed no ability to convert pyruvate into glucose (Fig. 8). No phosphoenolpyruvate carboxykinase (EG. 4.1.1.32) activity could be found in placenta homogenates.

The possibility of the presence of gluconeogenesis in the rat fetus has been investigated in vivo. Intraperitoneal injections of pyruvate- $2-{ }^{14} \mathrm{C}$ and glycerol- $1,3-{ }^{14} \mathrm{C}$ were given to 1-day-old newborns, prematures (fetuses which were delivered 1 day prior to term), and fetuses in situ. The l-day-old newborns do convert both substrates to glucose, with averages of 50 and $70 \%$, respectively, of serum radioactivity being due to glucose $-{ }^{14} \mathrm{C}$, thus proving the validity of this way of investigating gluconeogenesis in vivo. The prematures did not synthesize glucose from pyruvate, but glucose synthesis occurred from glycerol, an average of $70 \%$ of serum radioactivity being due to glucose${ }^{14} \mathrm{C}$. The fetuses which were injected in situ did not synthesize glucose from either one of the two substrates. These experiments would seem to exclude fetal gluconeogenesis as the source of fetal blood glucose in the constant infusion experiments mentioned above.

Fetal hepatic glycogenolysis can account only in part for the higher dilution of the specific activity of glucose in the fetus after a 16-hr fast. The data in Table I show that at the time when the constant infusions were started (after a 16-hr fast) the fetal liver glycogen concentration had diminished from $11 \mathrm{l} \pm 5$ to $82 \pm 10 \mathrm{mg} / \mathrm{g}$ wet weight, as compared to the fed state. This decrease is too small to account for the higher fetal than maternal dilution of blood glucose specific activity, or for the maintenance of unchanged blood glucose levels in the fetus after a 16-hr fast (86 \pm 8 and $92 \pm 11 \mathrm{mg} / 100 \mathrm{ml}$, respectively).

An unknown mechanism leading to the privileged situation of the fetus with regard to blood glucose levels during a fasting state must be postulated.

\section{Speculation}

Gluconeogenesis is inactive in the rat fetus, as investigated by in vivo and in vitro methods. Fetal hepatic glycogenolysis cannot be the only source of fetal blood glucose during fasting. 
The factors leading to the privileged situation of the fetus with regard to blood glucose levels during a fasting state are unknown. The study of these factors must consider the rates of placental transport, the rate of glucose utilization in the fetal and maternal compartment, and the role of fetal and maternal glycogen stores in contributing blood glucose during a fasting period.

\section{Introduction}

Measurements of the activities of the four gluconeogenetic key enzymes in fetal rat liver have shown that pyruvate carboxylase, fructose-1,6-diphosphatase, and glucose-6-phosphatase are detectable, whereas no soluble phosphoenolpyruvate carboxykinase activity can be found during gestation [1,2, 13]. The lack of activity of this soluble enzyme is thought to explain the nonexistence of gluconeogenesis in the rat fetus, as shown by studies on the incorporation of pyruvate- ${ }^{-14} \mathrm{C}$ into glucose by liver slices $[2,3,12,13]$. This activity becomes measurable after birth and increases 25-fold within 2 days $[2,13]$. Concomitantly, overall gluconeogenesis becomes operative, as evidenced by incorporation of radioactive pyruvate into glucose by liver slices [12]. Cytosol phosphoenolpyruvate carboxykinase activity can be induced in fetal liver through the injection of glucagon into the fetus [4]. However, in vivo experiments with anesthetized animals under these conditions indicate no gluconeogenesis. Philippidis and Ballard [9] were not able to detect gluconeogenesis in fetuses by injecting radioactive lactate, pyruvate, or aspartate intraperitoneally and by measuring incorporation of the radioactivity into liver glucose. In contrast, Goodner and Thompson [6] concluded, from calculations based upon the finding of similar glycogen concentrations in fasted and glucose-loaded rat fetuses, that the gluconeogenetic pathway had to be operative in the rat fetus, since the fasting fetuses were maintaining normal blood glucose levels without showing a significant glycogenolysis.

We have carried out both in vivo and in vitro studies in an attempt to show gluconeogenesis in the rat fetus. We have reproduced the constant infusion studies of Goodner and Thompson [6] by following the specific activities of both uniformly labeled glucose- ${ }^{14} \mathrm{C}$ and glucose-2- ${ }^{3} \mathrm{H}$ in maternal and fetal serum several times during the course of the infusion of radioactive glucose. We also have injected radioactive gluconeogenetic precursors into fetuses, prematures, and newborns and determined the glucose production. The origin of fetal blood glucose was further investigated by measurements of hepatic glycogen concentrations prior to and after a fasting period.

\section{Materials and Methods}

\section{In Vivo Experiments}

Constant infusion of radioactive glucose. SpragueDawley rats late in pregnancy (from the 18th to the 21st day) were anesthetized by ip injection of Diabutal [14], $60-80 \mathrm{mg} / \mathrm{kg}$, after a $16-\mathrm{hr}$ fast. The inguinal artery and vein were prepared and a catheter [15] was inserted into each vessel. A priming dose of $0.5 \mu \mathrm{Ci}$ uniformly labeled glucose- ${ }^{-14} \mathrm{C}$ and $2 \mu \mathrm{Ci}$ glucose- $2-{ }^{-3} \mathrm{H}$ in $0.4 \mathrm{ml}$ saline was administered. Then, a mixture consisting of $20 \mu \mathrm{Ci}$ uniformly labeled glucose ${ }^{-14} \mathrm{C}$ in $0.3 \mathrm{ml}$ water, $100 \mu \mathrm{Ci}$ glucose- $2-{ }^{3} \mathrm{H}$ in $0.2 \mathrm{ml} 95 \%$ ethanol [16], $0.1 \mathrm{ml}$ heparin, and $0.2 \mathrm{ml}$ saline was infused at a constant rate [17] into the vein, in order to establish a steady state specific activity in the maternal blood. The rate of infusion delivered $0.09 \mu \mathrm{Ci}$ glucose- ${ }^{14} \mathrm{C}$ and $0.44 \mu \mathrm{Ci}$ glucose-2- ${ }^{-3} \mathrm{H}$ per min. Some experiments were carried out with uniformly labeled glucose- ${ }^{14} \mathrm{C}$ alone. Maternal blood was sampled at different time intervals through the arterial catheter. Fetal blood was collected by excising two to four fetuses (depending on their size), wiping them dry, cutting the chest, and then collecting the outflowing blood into a micropipette. The fetal blood samples were pooled.

After immediate centrifugation, an aliquot of the serum $(0.1 \mathrm{ml})$ was used for glucose determination by the glucose oxidase method [18] after protein precipitation according to Somogyi. The other aliquot of serum was deproteinized with $5 \% \mathrm{Ba}(\mathrm{OH})_{2}$ and $5 \%$ $\mathrm{ZnSO}_{4}$ in quantities yielding a $\mathrm{pH}$ of 7.1 . The glucose was separated from charged metabolites, e.g., pyruvate, by running the supernatant through a small column ( 5 $\mathrm{mm}$ in internal diameter) that contained $0.5 \mathrm{ml}$ Dowex I resin [19]. Four fractions of $4 \mathrm{ml}$ each were collected: the first two were eluted with water, the next two with $0.1 \mathrm{~N} \mathrm{HCl}$. The aqueous fractions were lyophilized in order to eliminate ${ }^{3} \mathrm{H}_{2} \mathrm{O}$ that had been formed, redissolved in $1 \mathrm{ml}$ water, and then counted in $10 \mathrm{ml}$ liquid scintillation fluid containing dioxane, naphthalene, POP, and POPOP. The fractions eluted with $0.1 \mathrm{~N}$ $\mathrm{HCl}$ were similarly counted. This chromatographic separation of glucose proved to be reasonable: only up 
to $2 \%$ of the counts of the charged metabolites contaminated the aqueous fractions. This value was calculated after appropriate corrections for quenching were made. Radioactivity in the aqueous fractions was proved to be due to glucose by incubating an aliquot of these fractions with glucose oxidase and then rechromatographing the incubation mixture on the anion exchange resin. Radioactivity could now be eluted only with $\mathrm{HCl}$, showing that there was only glucose in the aqueous fractions. The specific activity of glucose was then calculated as follows:

$$
\frac{\text { cpm as glucose } / 0.1 \mathrm{ml} \text { serum }}{\mathrm{mg} \text { glucose } / 0.1 \mathrm{ml} \text { serum }}
$$

Intraperitoneal injections of pyruvate-2-14C or glycerol-1,3-14C into fetuses, prematures, and full term newborns. The fetuses which were injected in situ were 2 days before birth. The prematures were delivered 1 day prior to term. The full term newborns were 12-36 hr old at the time of the investigations.

After anesthesia, the maternal abdomen was opened and the fetuses of one horn were injected ip with either $5 \mu \mathrm{Ci}$ pyruvate- $2-{ }^{14} \mathrm{C}$ or $7 \mu \mathrm{Ci}$ glycerol-1, $3-{ }^{-14} \mathrm{C}$, dissolved in $0.05 \mathrm{ml}$ saline. The fetuses of the other horn were not injected. Blood was sampled as described at specific times. The deproteinized serum was chromatographed as mentioned. No lyophilization had to be carried out in this group of experiments. Radioactivity was measured as described. The radioactive glucose formed from pyruvate appeared in the aqueous fractions, whereas pyruvate had to be eluted with HCI. The amount of radioactive glucose was calculated as the percentage of total serum radioactivity appearing in the water effluent. Again, appropriate corrections for quenching were made.

For the determination of the glucose synthesized from glycerol, the aqueous fractions from chromatography had to be incubated with glucose oxidase. Then, rechromatography was carried out on columns $12 \mathrm{~mm}$ in internal diameter that contained $5.5 \mathrm{ml}$ Dowex 1 resin [19]. Two fractions of $4 \mathrm{ml}$ each were eluted with water, two with $0.1 \mathrm{~N} \mathrm{HCl}$. The fractions which were eluted with $\mathrm{H}_{2} \mathrm{O}$ contained the glycerol, and the ones eluted with $\mathrm{HCl}$ contained gluconic acid as a measure of the glucose which had been synthesized from glycerol-1, $3{ }^{-14} \mathrm{C}$. The amount of radioactive glucose synthesized from glycerol was calculated as the percentage of total serum radioactivity appearing as gluconic acid.

The prematures were delivered by cesarean section 1 day prior to term. They were kept under a regular lamp and injected intraperitoneally within $45 \mathrm{~min}$ of delivery.

\section{In Vitro Experiments}

Incubation of slices of liver and placenta with pyruvate-2-14 $C$. We used placentas and livers of animals which were $1-2$ days prior to term.

Immediately after removal, placentas and livers were dropped into ice-cold Krebs-Henseleit bicarbonate buffer [8]. The organs were then sliced and kept in the cold buffer. Incubations were carried out in a Dubnoff metabolic shaker at $37^{\circ}$ for 1 and $2 \mathrm{hr}$ in a $95 \%$ $\mathrm{O}_{2}-5 \% \mathrm{CO}_{2}$ atmosphere. Three groups of incubation mixtures were used for the slices of placentas. They all consisted of bicarbonate buffer containing $\mathrm{Na}$ pyruvate- $2-{ }^{14} \mathrm{C}$ ( $4 \mathrm{ml}$ bicarbonate buffer containing $0.8 \mu \mathrm{Ci}$ radioactive pyruvate per beaker). The three groups differed in the concentration of cold $\mathrm{Na}$ pyruvate: 0.15 м, $0.08 \mathrm{M}$, or $0.015 \mathrm{M}$. For liver slices, $0.15 \mathrm{~m}$ pyruvate was used and the incubation lasted for $1.5 \mathrm{hr}$. The slices were then removed from the incubation mixtures, and the whole incubation medium was chromatographed through the large columns mentioned above. Three fractions of $4 \mathrm{ml}$ each were eluted with water and two with $0.1 \mathrm{~N} \mathrm{HCl}$. The aqueous fractions contained the glucose. One milliliter of each fraction was counted as described. The recovery index for glucose was found to be practically $100 \%$.

Determination of glycogen concentrations. The concentration of glycogen in maternal and fetal livers and in placentas was determined after purification of glycogen with $60 \% \mathrm{KOH}(2 \mathrm{ml}$ containing $300-500 \mathrm{mg}$ fresh tissue were placed in a boiling water bath for 1 hr) and $75 \%$ ethanol $(10 \mathrm{ml}$ of which were added to the mixture containing the tissue; the mixture was then brought to a boil. The supernatant was poured off, $10 \mathrm{ml}$ ethanol were added, and the mixture was then centrifuged). This last procedure was repeated for a total of four times, the final precipitate being redissolved in $2 \mathrm{ml} \mathrm{H}_{2} \mathrm{O}$. The concentration of the purified glycogen was then determined by a colorimetric assay with anthrone (according to Seifter et al. [10]).

\section{Results}

\section{In Vivo Experiments}

Maternal and fetal blood glucose concentrations at different gestational ages. The maternal and fetal blood glucose concentrations that were measured during the infusions of radioactive glucose into seven animals are plotted in Figure 1: the younger the fetus, the higher the ratio of maternal to fetal serum glucose concentration. By the time of birth, fetal serum glu- 


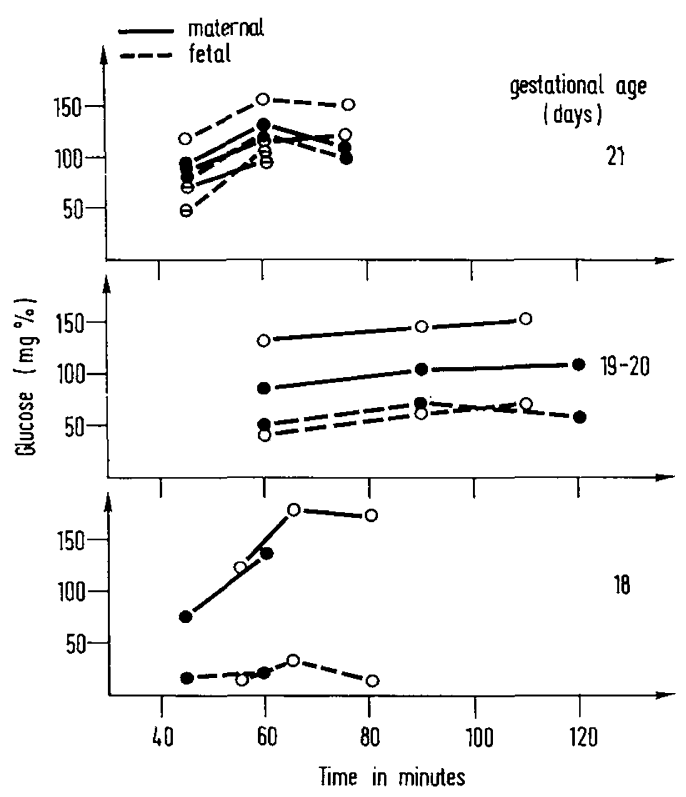

lig. 1. Maternal and fetal serum glucose concentrations at three different gestational ages during the constant infusion of glucose${ }^{14} \mathrm{C}$ and of glucose- $2-{ }^{3} \mathrm{H}$ of high specific activity. Each point represents one single determination. The blood of two, three, or four fetuses (depending on fetal size) was pooled for the determinations of the points in Figures $I$ to 3.

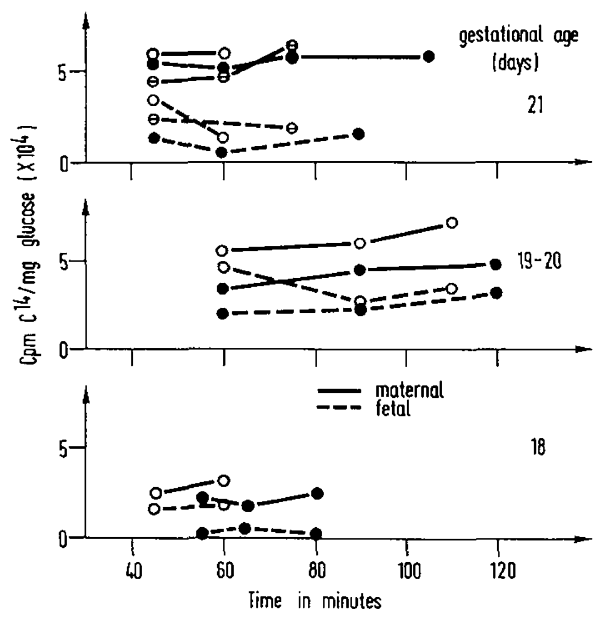

Fig. 2. Specific activity of glucose $-{ }^{14} \mathrm{C}$ in maternal and fetal serum at three different gestational ages, during the constant infusion of glucose $-{ }^{14} \mathrm{C}$ and of glucose $-2{ }^{3} \mathrm{H}$ of high specific activity.

cose concentrations have reached maternal values. Hematocrit values did not vary significantly between the three letal age groups.

Specific activity of maternal and fetal blood glucose during constant infusions of radioactive glucose. Figures 2 and 3 show that the specific activity of glucose as determined by using glucose- ${ }^{14} \mathrm{C}$ alone in two expeximents or glucose- ${ }^{-14} \mathrm{C}$ and glucose- $2-{ }^{3} \mathrm{H}$ in five dou- ble labeling experiments) was, at all times measured during the infusions, two to four times higher in the maternal than in the fetal blood. This pattern is independent of gestational age. Two more double labeling experiments and two more experiments with glucose${ }^{14} \mathrm{C}$ alone, distributed over the different gestational ages, gave the same results.

Injection of pyruvate-2-14C into fetuses, prematures, and newborns. Figure 4 shows that no glucose has been synthesized from pyruvate by the fetuses. In contrast, the mother converts this precursor, which she receives through the placenta, at a very fast rate. Five minutes after the injections, $10 \%$ of the radioactivity

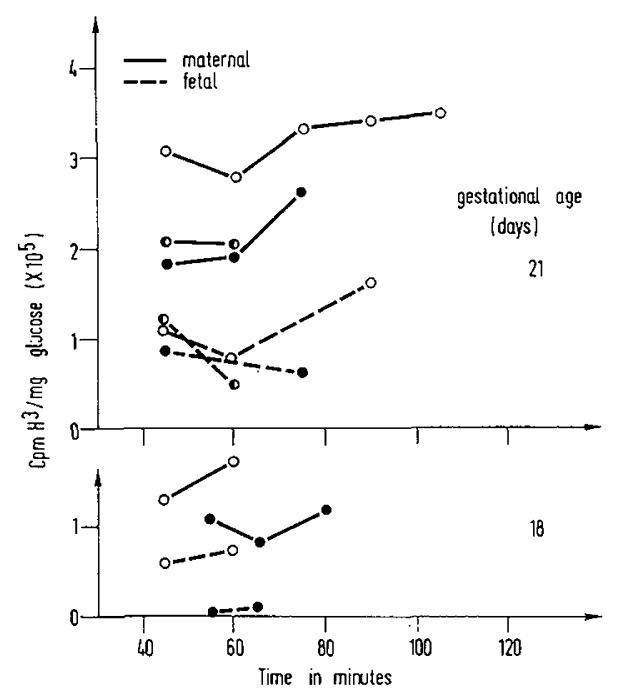

Fig. 3. Specific activity of glucose- $2-{ }^{3} \mathrm{H}$ in maternal and fetal serum at two different gestational ages during the constant infusion of glucose-2- ${ }^{3} \mathrm{H}$ and uniformly labeled glucose $-{ }^{14} \mathrm{C}$ of high specific activity.

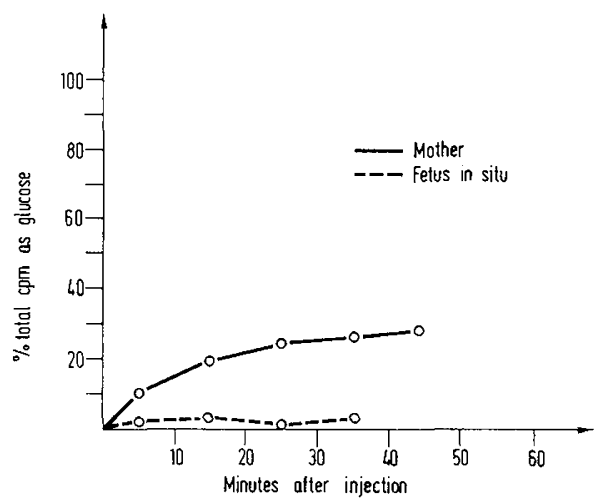

Fig. 4. Percentage of total radioactivity in the maternal and fetal serum, due to glucose ${ }^{-14} \mathrm{C}$, after the injection of $5 \mu \mathrm{Ci}$ pyruvate$2{ }^{24} \mathrm{C}$ into each of four fetuses in situ. This experiment is representative of three additional experiments; a total of 19 fetuses was investigated. 


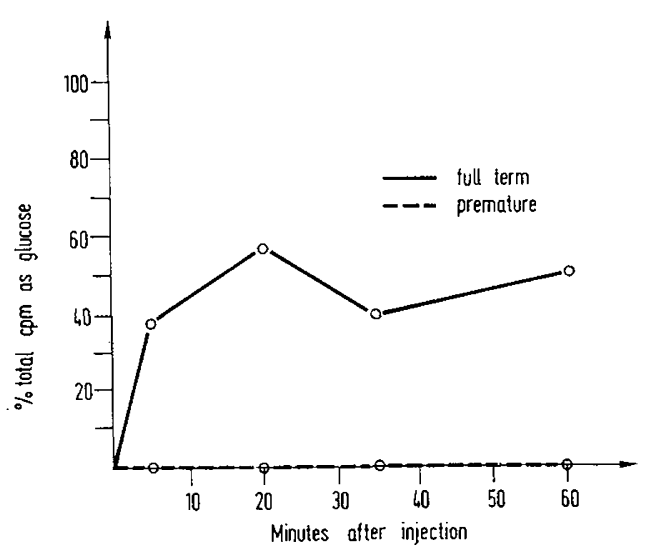

Fig. 5. Percentage of total radioactivity as glucose $-{ }^{34} \mathrm{C}$ in the serum of eight full term newborns investigated 12 to $36 \mathrm{hr}$ after birth and of eight prematures, after injection of $5 \mu \mathrm{Ci}$ pyruvate$2 \cdot{ }^{14} \mathrm{C}$. Each point represents the mean of two determinations on pooled blood from two animals. A total of 16 full term newborns and 16 prematures was investigated.

in the maternal serum is already due to glucose- ${ }^{-14} \mathrm{C}$. The low glucose- ${ }^{14} \mathrm{C}$ counts in the fetus are probably due to placental crossing of glucose- ${ }^{-14} \mathrm{C}$, since the same percentage was found in the noninjected fetuses (not shown in the figure).

Figure 5 shows that the intraperitoneal injection of pyruvate- $2-{ }^{14} \mathrm{C}$ in the full term newborn does lead to the synthesis of glucose- ${ }^{14} \mathrm{C}$. Approximately $50 \%$ of the injected pyruvate has been converted to glucose. This proves the validity of this method for investigating gluconeogenesis in vivo.

Prematures are unable to convert pyruvate to glucose.

Injection of glycerol-1, $3-{ }^{14} \mathrm{C}$ into fetuses, prematures, and newborns. Five minutes after the ip injection of glycerol-1, $3-{ }^{-14} \mathrm{C}$ into fetuses in situ, $54 \%$ of the radioactivity in maternal serum was due to glucose- ${ }^{14} \mathrm{C}$ (Fig. 6). Five minutes later, this value had risen to $64.5 \%$. The injected fetuses showed no conversion after $5 \mathrm{~min}$; $1 \%$ of the total counts was due to glucose ${ }^{14} \mathrm{C}$ at that time. Ten minutes after the injection, $9 \%$ of the total counts was due to glucose $-{ }^{14} \mathrm{C}$. We interpret this time-dependent increase as being caused by placental crossing of glucose from the mother, since $12 \%$ of the total counts in the serum of the fetuses of the other horn (which had not been injected) was due to glucose ${ }^{14} \mathrm{C}$. For technical reasons, it was not possible to pursue this group of experiments, which was comprised of three more experiments with a total of 17 fetuses, for longer than 10 min.

After ip injection of radioactive glycerol into full term newborns, radioactive glucose could be found in the serum of these animals. An average of $70 \%$ of the total counts were counts as gluconic acid (Fig. 7).

Figure 7 also shows that fetuses which were delivered surgically 1 day prior to term (prematures) and were injected within $45 \mathrm{~min}$ of delivery converted the glyc-

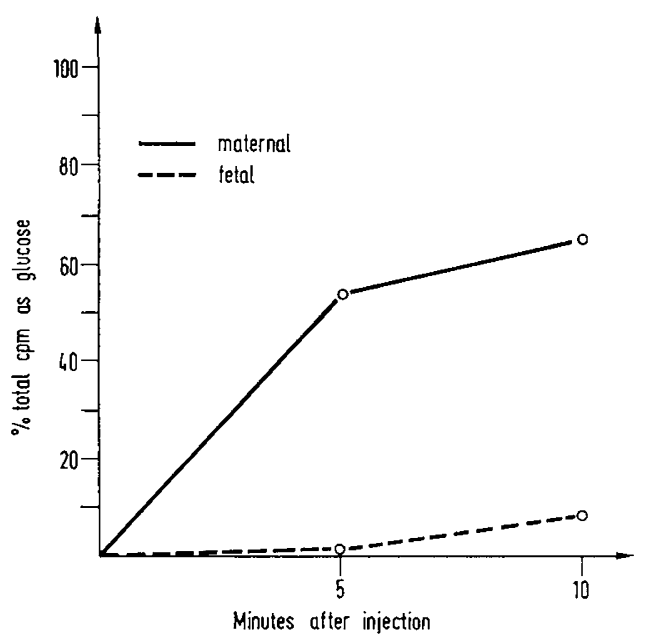

Fig. 6. Percentage of the total radioactivity as glucose ${ }^{-4} \mathrm{C}$ in the maternal and the fetal serum after injection of $7 \mu \mathrm{Ci}$ glycerol$1,3{ }^{14} \mathrm{C}$ into each of six fetuses in situ. Each point represents the mean of two determinations on pooled blood from three fetuses and one determination on each maternal blood sample.

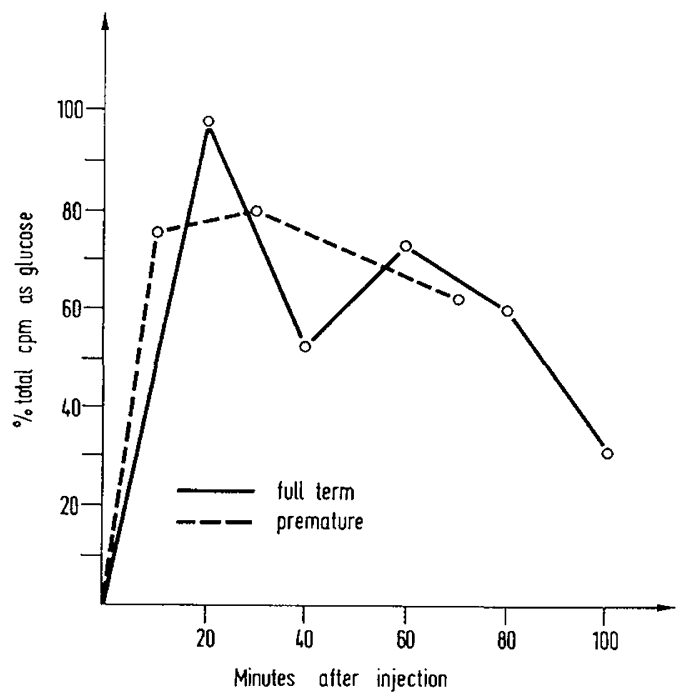

Fig. 7. Percentage of total radioactivity as glucose $-{ }^{14} \mathrm{C}$ in the serum of five full term newborns investigated 12 to $36 \mathrm{hr}$ after birth and of six prematures, after injection of $7{ }_{\mu} \mathrm{Ci}$ glycerol$1,3-{ }^{14} \mathrm{C}$. For the prematures, each point represents the mean of two determinations on pooled blood from two animals. For the full term newborns, each point represcnts a single determination on one animal. A total of 12 full term newborns and 14 prematures was investigated. 
erol to glucose. The conversion rate was of the same order of magnitude as that for newborns.

\section{In Vitro Experiments}

Incubation of slices of maternal, fetal, and newborn liver, as well as placenta, with pyruvate-2-14 $\mathrm{C}$. Figure 8 shows the results of the incubation of slices of placenta, maternal liver, fetal liver, and newborn liver with $\mathrm{Na}$ pyruvate- $2-{ }^{-14} \mathrm{C}$. It can be seen that maternal and newborn liver slices converted the pyruvate into glucose, whereas slices of fetal liver (1-2 days prior to term), as well as slices of placenta (the latter assayed at three different substrate concentrations) did not. Furthermore, we were not able to demonstrate an activity of phosphoenolpyruvate carboxykinase in homogenates of placenta, by measuring the incorporation of ${ }^{14} \mathrm{CO}_{2}$ into malate according to Chang et al. [5].

Liver and placenta glycogen concentrations and serum glucose levels of fed and fasted mothers and fetuses. Table I gives the glycogen concentrations in

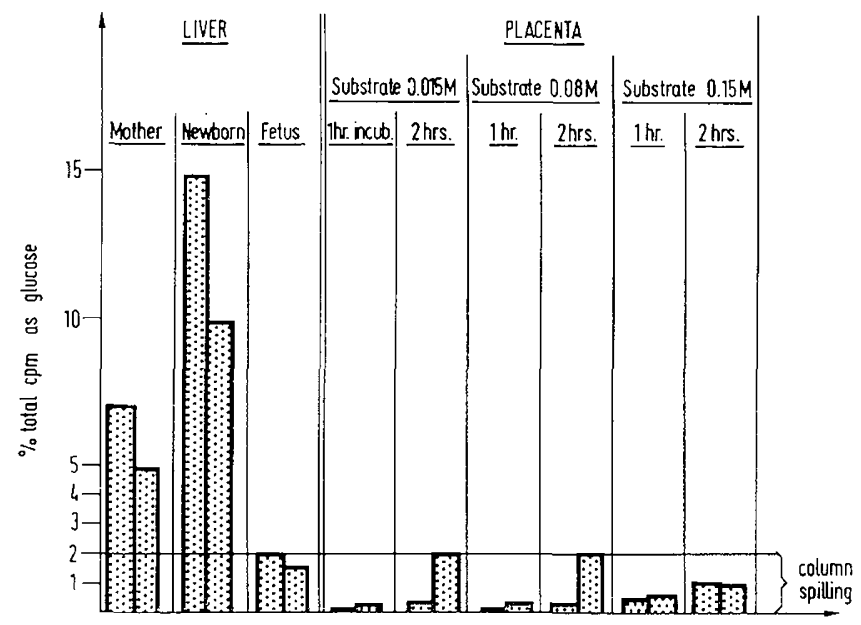

Fig. 8. Conversion of pyruvate- $-2{ }^{-14} \mathrm{C}$ to glucose $-{ }^{-14} \mathrm{C}$ by slices of matemal, newborn (at least $12 \mathrm{hr}$ old), and fetal livers and of placentas. The results are expressed as the precentage of total radioactivity in the incubation media which is due to glucose $-{ }^{14} \mathrm{C}$. Each bar represents the value for one slice. For liver slices, $0.15 \mathrm{M}$ $\mathrm{Na}$ pyruvate was used and the incubations lasted $1.5 \mathrm{hr}$. the placenta and the liver of four normally fed mothers and of eight of their fetuses and of four fasted mothers and of eight of their fetuses, as determined on the 21st day of gestation. It can be seen that after a 16-hr fast, the maternal liver was almost completely depleted of glycogen (fed: $19 \pm 3 \mathrm{mg}$ glycogen $/ \mathrm{g}$ wet weight; fasted: $0.3 \pm 0.1 \mathrm{mg}$ glycogen $/ \mathrm{g}$ wet weight). The glycogen concentration of fetal livers was also diminished, but to a lesser extent (fed: $111 \pm 5 \mathrm{mg}$ glycogen/g wet weight; fasted: $82 \pm 10 \mathrm{mg}$ glycogen $/ \mathrm{g}$ wet weight).

The placental glycogen concentrations are too low to be of physiologic importance since the total placentas weigh only twice as much as the whole fetal liver (averages of $5.95 \mathrm{~g}$ versus $3.20 \mathrm{~g}$ ). Furthermore, the glycogen concentrations in the placenta do not vary significantly.

Concomitantly with the depletion of maternal liver glycogen, the maternal blood glucose levels drop during the fasting period (from $91 \pm 2$ to $66 \pm 3 \mathrm{mg} / 100$ $\mathrm{ml}$ ). At the time of the determinations, after the $16-\mathrm{hr}$ fast, fetal blood glucose levels were unchanged with respect to the levels in the offspring of fed animals.

\section{Discussion}

We have been able to confirm the findings of Goodner and Thompson [6]: if radioactive glucose is infused at a constant rate into a fasting, anesthetized SpragueDawley rat in the last 3 days of gestation, the fetal specific activity of the blood glucose is always two to four times lower than the maternal. This is true if uniformly labeled glucose- $-{ }^{14} \mathrm{C}$ or glucose-2- ${ }^{3} \mathrm{H}$ is used. These results cannot be due to recycling of the label when glucose- $2-{ }^{3} \mathrm{H}$ is used, since this label is lost at the phosphofructoisomerase step. Two requirements must be met in order for these results to be interpretable. First, the specific activity of glucose in the maternal serum must reach a steady state during the infusion. This requirement has been fullfilled in our experiments. Secondly, a free passage of glucose through the placenta is required. As reviewed by Widdas [11], how-

Table $X$. Concentrations of glycogen in maternal liver, fetal liver, and placenta and of glucose in serum of fed and fasted animals on the 21st day of gestation ${ }^{1}$

\begin{tabular}{lccccc}
\hline & \multicolumn{3}{c}{ Glycogen, $\mathrm{mg} / \mathrm{g}$ wet weight } & & \multicolumn{2}{c}{ Serum glucose, mg/100 ml } \\
\cline { 2 - 4 } & Maternal liver & Fetal liver & Placenta & & Maternal \\
\hline Fed & $19 \pm 3$ & $111 \pm 5$ & $1.2 \pm 0.3$ & $91 \pm 2$ & $86 \pm 8$ \\
Fasted (16 hr) & $0.3 \pm 0.1$ & $82 \pm 10$ & $1.8 \pm 0.3$ & $66 \pm 3$ & $92 \pm 11$ \\
\hline
\end{tabular}

${ }^{1}$ The results are given as the mean \pm SEM of the measurements in four maternal livers, eight fetal livers, and eight placentas and as the mean \pm SEM of four blood glucose determinations. 
ever, glucose is believed not to pass the placenta freely, but rather by a mechanism of "facilitated diffusion." In Figure I we show the considerable variation in fetal and maternal serum glucose concentrations at 18 days of gestation. The fetal values increase more and more as the gestation proceeds until they reach the maternal values at the 20 th-21st day. These findings also indicate that some sort of deviation from free diffusion might be present for glucose in the placenta. This deviation itself might also be subject to changes as the gestation proceeds and the placenta grows older. Despite this, it must be emphasized that the difference between maternal and fetal specific activity of glucose is the same at all gestational ages investigated, even though the fetal blood glucose concentration increases up to maternal levels as gestation proceeds.

In order to understand what mechanism was responsible for the higher fetal than maternal dilution of the specific activity of serum glucose, or, in other words, what the source of cold glucose in the fetal serum was, the following experiments were carried out.

We concentrated on gluconeogenesis first: the ability of the fetus to convert pyruvate and glycerol into glucose was tested. Intraperitoneal injections of radioactive pyruvate or glycerol into l-day-old newborns allowed us to recover radioactive glucose in the serum (Figs. 5 and 7). This indicates the validity of this method for the in vivo detection of a functioning gluconeogenetic pathway; in vitro studies have given evidence for the activity of gluconeogenesis at this stage, since all gluconeogenic key enzymes are active in the liver of rats at this time. Pyruvate failed to be converted to glucose by fetuses which were delivered surgically I day prior to term, injected, and studied within 2 hr of delivery (Fig. 5), nor was pyruvate converted to glucose by fetuses which were injected in situ 2 days betore birth (Fig. 4).

Pyruvate having failed to prove its role as gluconeogenetic precursor in the fetus, we carried out the same kind of in vivo experiments with glycerol-1, $3{ }^{-14} \mathrm{C}$, in order to see if the gluconeogenetic pathway was functioning in the fetuses above the triose step. Glycerol was not converted to glucose when it was administered to fetuses in situ (2 days before birth; Fig. 6). This figure shows a time-dependent increase in counts as glucose in the serum of the fetuses. We interpret this time-dependent increase as being caused by placental crossing of radioactive glucose from the mother to the fetuses, since the same increase was found in the fetuses which were not injected. In contrast to pyruvate, glycerol was converted to glucose by fetuses which had been delivered surgically 1 day prior to term (Fig. 7). The increase in glucose counts in the serum of the prematures is of the same order of magnitude and rate as the increase in the serum of the full term newborns. The fetuses which were studied in situ were 1 day younger than those prematurely delivered. This fact might indicate that gluconeogenesis from glycerol develops during the last few days of gestation, since the prematures (birth minus 1 day) did synthesize glucose from glycerol, whereas the fetuses in situ (birth minus 2 days) did not. It is in fact known [7] that low activities of glycerokinase become measurable in fetal rat liver at the 21st day of gestation. It is also possible that premature delivery itself might trigger gluconeogenesis from glycerol, as premature delivery has been shown to trigger gluconeogenesis from pyruvate by promoting the appearance of phosphoenolpyruvate carboxykinase activity in liver [12]. But, still, a gluconeogenetic pathway with glycerol as precursor, which became operative during the last day of gestation, or after premature delivery, could not explain the results of the constant infusion experiments carried out in earlier gestation.

Since the in vivo experiments indicated that gluconeogenesis is not operative in the intact fetus, the gluconeogenetic capacity of the placenta was tested. In vitro studies with placenta slices showed no ability of the placenta to synthesize glucose from pyruvate (Fig. 8). An activity of phosphoenolpyruvate carboxykinase could not be found in homogenates of placenta.

Recently, Ballard and Philippidis [4] have shown that the activity of the missing gluconeogenetic key enzyme phosphoenolpyruvate carboxykinase in the fetal liver can be activated by glucagon. This mechanism could be operative during fasting. This activation, however, takes place only in vitro. Anesthetized animals do not show this phenomenon. The dilution of the specific activity of blood glucose which was found by Goodner and Thompson [6] and confirmed by us therefore cannot be due to this activation. Whether this mechanism would be operative in the nonanesthetized animal in vivo is not known. However, our experiments with liver slices of fetuses of fasted, not anesthetized mothers have shown no gluconeogenesis (Fig. 8). The glucagon secretion during fasting therefore does not seem to activate phosphoenolpyruvate carboxykinase in the fetus.

According to the results shown in Table I, fetal liver glycogen appears to be in dynamic equilibrium with the fetal blood glucose during a maternal fast. After a 16-hr fast, fetal livers contain about two-thirds of their 
original glycogen, and there is no drop in fetal serum glucose concentration. It can, however, be calculated that the drop in fetal liver glycogen concentration is too small and the glucose consumption in the fetus too high for fetal hepatic glycogenolysis to be the only responsible factor for the higher fetal than maternal dilution of the specific activity of blood glucose. This is at least true for the 21-day-old fetuses, which were the only ones to be studied in this regard.

Our experiments cannot offer an explanation for the higher fetal than maternal dilution of the specific activity of blood glucose during fasting. They confirm the privileged situation of the fetus with regard to blood glucose levels during a fasting state, as described by Goodner and Thompson [6]. They also confirm these authors' findings that fetal hepatic glycogenolysis alone cannot suffice to maintain high fetal blood glucose levels. However, they exclude gluconeogenesis as the source of fetal blood glucose during a fasting state.

\section{Summary}

Constant infusions of uniformly labeled glucose- ${ }^{-14} \mathrm{C}$ and glucose-2- ${ }^{3} \mathrm{H}$ into fasted, anesthetized SpragueDawley rats in the last 3 days of gestation lead to a lower specific activity of glucose in the fetal than in the maternal serum. The source of fetal blood glucose was investigated.

In vivo experiments, namely intraperitoneal injections of pyruvate-2-14 $\mathrm{C}$ and glycerol- $1,3-{ }^{14} \mathrm{C}$ into fetuses in situ and prematures, are indicative of the absence of gluconeogenesis in the rat fetus.

In vitro experiments with placenta slices which were incubated with radioactive pyruvate showed an inability of the placenta to synthesize glucose from pyruvate. Furthermore, no activity of the gluconeogenetic key enzyme phosphoenolpyruvate carboxykinase could be measured in placenta homogenates.

Measurements of glycogen concentrations in maternal livers, fetal livers, and placentas of fed animals and of animals which had been fasted for $16 \mathrm{hr}$ showed fetal liver glycogen to be in dynamic equilibrium with fetal blood glucose. However, fetal hepatic glycogenolysis alone cannot quantitatively account for the higher fetal than maternal dilution of blood glucose specific activity.

An unknown mechanism leading to the privileged situation of the fetus with regard to blood glucose levels during a fasting state must be postulated.

\section{References and Notes}

1. Ballaro, F. J.: Carbohydrate metabolism in fetal and neoCopyright (C) 1972 International Pediatric Research Foundation, Inc. natal liver. Ph.D. thesis, University of Western Australia (1964).

2. Ballard, F. J., and Hanson, R. W.: Phosphoenolypyruvate carboxykinase and pyruvate carboxylase in developing rat liver. Biochem. J., 104: 866 (1967).

3. Ballard, F. J., ANd Oliver, I. T.: Glycogen metabolism in embryonic chick and neonatal rat liver. Biochim. Biophys. Acta, 71:578 (1963).

4. Ballard, F. J., And Philmprois, H.: The development of gluconeogenic function in rat liver. In: H. D. Sölnig and B. Willms: Regulation of Gluconeogenesis. (Springer, Berlin, 1971).

5. Chang, H. C., and Lane, M. D.: The enzymatic carboxylation of phosphoenolpyruvate. II: Purification and properties of liver mitochondria phosphoenolpyruvate carboxykinase. J. Biol. Chem., 241: 2413 (1966).

6. Goodner, C. J., And Thompson, D. J.: Glucose metabolism in the fetus in utero: the effect of maternal fasting and glucose loading in the rat. Pediat. Res., 1: 443 (1967).

7. Hahn, P., ANo GreenberG, R. E.: The development of pyruvate kinase, glycerol kinase and phosphoenolpyruvate carboxykinase activities in liver and adipose tissue of the rat. Experientia, 24: 428 (1968).

8. Krebs, H. A., and Henseleit, K.: Untersuchungen über die Harnstoffbildung im Tierkoerper. Hoppe-Seyler's Z. Physiol. Chem., 2: 210: 33 (1932).

9. Philippidis, H., and Ballard, F. J.: The development of gluconeogenesis in rat liver. Biochem. J., 113: 651 (1969).

10. Seifter, S., Dayton, S., Novic, B., and Muntwyler, E.: The estimation of glycogen with the anthrone reagent. Arch. Biochem., 25: 191 (1950).

11. Widdas, W. F.: Transport mechanisms in the fetus. Brit. Med. Bull., 17: 107 (196l).

12. Yeunc, D., AND Oliver, I. T.: Development of gluconeogenesis in neonatal rat liver. Effect of premature delivery. Biochem. J., 105: 1229 (1967).

13. Yeung, D., Stanley, R. S., And Oliver, I. T.: Development of gluconeogenesis in neonatal rat liver. Effect of triamcinolone. Biochem. J., 105: 1219 (1967).

14. Diabutal, Diamond Company, Des Moines, Iowa.

15. Bardic Intracaths, size 1619-R, Bard, Inc., Murray Hill, N. J.

16. All isotopes were purchased from New England Nuclear Corporation, Boston, Mass. The specific activities of the radioactive compounds were sufficiently high, so that the contribution of the nonradioactive components was negligible. Specific activities were: glucose $-{ }^{14} \mathrm{C}, 77 \mu \mathrm{Ci} / \mathrm{mg}$; glucose- $2{ }^{3} \mathrm{H}, 1720$ $\mu \mathrm{Ci} / \mathrm{mg}$; pyruvate- $2-{ }^{14} \mathrm{C}$, $29 \mu \mathrm{Ci} / \mathrm{mg}$; glycerol-1, $3-{ }^{14} \mathrm{C}, \quad 125$ $\mu \mathrm{Ci} / \mathrm{mg}$.

17. Infusion pump, Compact Infusion Pump model no. 975, Harvard Apparatus Co., Inc., Dover, Mass.

18. Glucostat, Worthington Biochemical Corporation, Freehold, N. J.

19. Dowex 1 resin, purchased from BioRad as AG $1 \times 8,100-200$ mesh, was used as purchased in $\mathrm{Cl}^{-}$form for all chromatographies.

20. Supported in part by the National Institutes of Health Grant HD.02147.

21. Requests for reprints should be addressed to: Emilio Bossi, M.D., Universitaets-Kinderklinik, Inselspital, 3008 Berne, Switzerland.

22. Accepted for publication May 10, 1972. 